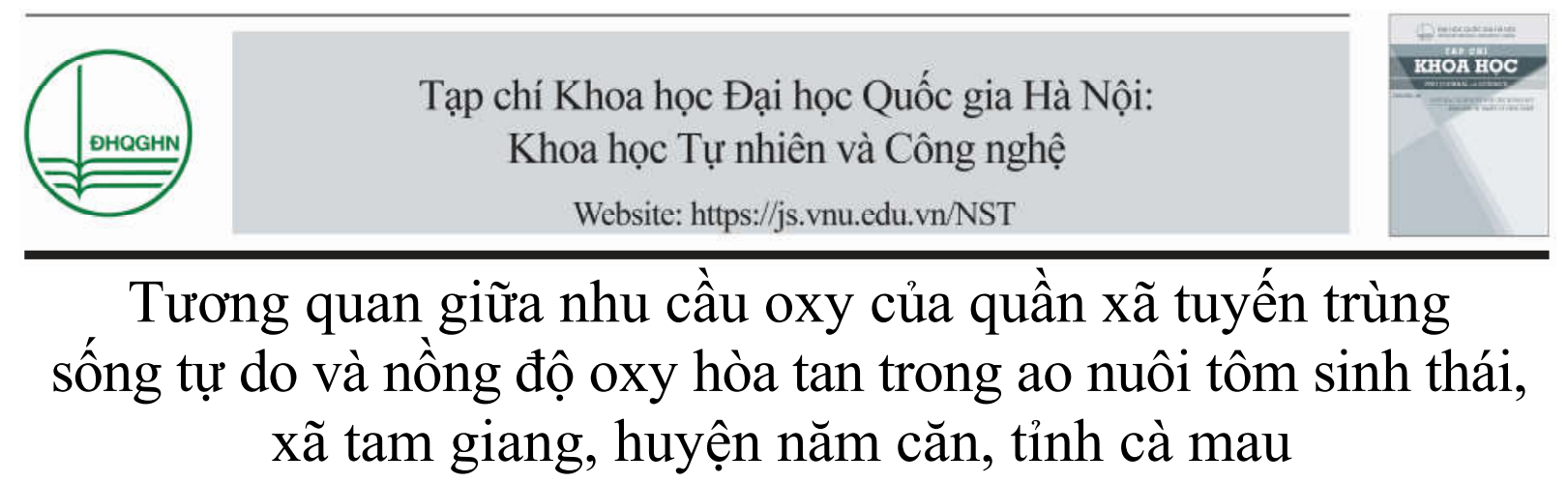

\author{
Trần Thành Thái ${ }^{1, *}$, Nguyễn Lê Quế Lâm ${ }^{1}$, \\ Nguyễn Thị Mỹ Yến ${ }^{1,2}$, Ngô Xuân Quảng ${ }^{1}$ \\ ${ }^{1}$ Viện Sinh học Nhiệt đới, Viện Hàn lâm Khoa học và Công nghệ Việt Nam \\ 85 Trần Quốc Toản, TP.Hồ Chí Minh, Việt Nam \\ ${ }^{2}$ Đai hoc Ghent, 281 Krijgslaan, S8, B-9000 Ghent, Bi \\ Nhận ngày 16 tháng 8 năm 2017 \\ Chỉnh sửa ngày 20 tháng 9 năm 2017; Chấp nhận đăng ngày 10 tháng 10 năm 2017
}

\begin{abstract}
Tóm tắt: Nồng độ oxy hòa tan (DO), tổng sinh khối khô trung bình (SKKTB) và nhu cầu oxy (Ox) của quần xã tuyến trùng sống tự do (QXTT) trong các ao nuôi tôm sinh thái (NTST) xã Tam Giang, huyện Năm Căn, tỉnh Cà Mau được khảo sát trong 3 đợt: tháng 3, 7 và 11 năm 2015 (tương ứng với mùa khô, chuyển mùa và mùa mưa ở miền $\mathrm{Nam}$ Việt $\mathrm{Nam}$ ). Kết quả ghi nhận $\mathrm{DO}$ nằm trong giới hạn cho phép nhưng hầu hết các giá trị đều tập trung ở giới hạn dưới. Tổng sinh khối khô trung bình của QXTT từ 24,77 đến $937,04 \mu \mathrm{gC} / 10 \mathrm{~cm}^{2}$ và $\mathrm{Ox}$ dao động từ 3467,39 đến $64288,50 \mathrm{nlO} 2 / \mathrm{ngày} / 10 \mathrm{~cm}^{2}$. Giá trị $\mathrm{SKKTB}$ và Ox khá cao so với các nghiên cứu khác trên thế giới. Ngoài ra, nghiên cứu cũng ghi nhận mối tương quan nghịch có ý nghĩa thống kê giữa $\mathrm{DO}$ và $\mathrm{Ox}$. Chứng tỏ quá trình hô hấp và chuyển hóa vật chất của $\mathrm{QXTT}$ có ảnh hưởng đến DO trong ao NTST.
\end{abstract}

Tư khóa: Ao tôm sinh thái, Cà Mau, nhu cầu oxy, oxy hòa tan, quần xã tuyến trùng, sinh khối

\section{Mở đầu}

Nuôi tôm công nghiệp là một trong những nguyên nhân làm xóa xổ một diện tích lớn rừng ngập mặn ven biển, đặc biệt là ở vùng đồng bằng sông Cửu Long [1,2]. Để giải quyết hiện tượng trên, mô hình NTST ra đời. Nuôi tôm

\footnotetext{
*Tác giả liên hệ. ĐT.: 84-

Email: thanhthai.bentrect@gmail.com

https://doi.org/10.25073/2588-1140/vnunst.4723
}

sinh thái là hình thức nuôi tôm trong rừng ngập mặn, tận dụng tôm giống và thức ăn tự nhiên và hoàn toàn không có sự can thiệp của con người [3]. Mặc dù đang mở rộng diện tích và phát triển mạnh nhưng có rất ít các công trình nghiên cứu trong ao NTST, đặc biệt là nghiên cứu về mối quan hệ giữa các thành phần hữu sinh và vô sinh trong ao tôm [4].

Nhóm động vật đáy không xương sống cỡ trung bình, đặc biệt là nhóm tuyến trùng sống tự 
do được xem như nguồn thức ăn tự nhiên cho tôm $[5,6]$. Ngoài ra, thông qua các hoạt động trao đồi chất, nhóm tuyến trùng còn đóng vai trò trong việc phân hủy và chuyển hóa vật chất nền đáy [7]. Một số nghiên cứu ở vùng cận nhiệt và ôn đới đã ước tính lượng oxy tuyến trùng cần cho quá trình trao đổi chất, lượng oxy này khác nhau theo mùa và theo vùng địa lý [8]. Vậy lượng oxy tuyến trùng dùng cho quá trình hô hấp có ảnh hưởng đến nồng độ oxy hòa tan (DO - Dissolved Oxygen) trong ao tôm hay không là vấn đề còn chưa nghiên cứu.

Cho nên mục tiêu của nghiên cứu này là (i) Thu thập số liệu về nồng độ $\mathrm{DO}$; sinh khối và nhu cầu oxy cần thiết cho quá trình trao đổi chất của QXTT trong ao tôm theo không gian và thời gian, (ii) Ghi nhận mối tương quan giữa $\mathrm{DO}$ và nhu cầu oxy của $\mathrm{QXTT}$.

\section{Phương pháp nghiên cứu}

\subsection{Khu vực nghiên cúu}

Cà Mau là tỉnh có năng suất và diện tích nuôi tôm lớn nhất cả nước (MARD, 2016) [9]. Hầu hết diện tích nuôi tôm tập trung ở các huyện như Năm Căn, Đầm Dơi và Cái Nước. Nằm ven sông Cửa Lớn, xã Tam Giang, huyện Năm Căn được xem như khu vực có nhiều điều kiện thuận lợi để phát triển mô hình nuôi tôm NTST.

Quần xã tuyến trùng và nồng độ oxy được khảo sát trong 8 ao NTST xã Tam Giang, huyện Năm Căn, tỉnh Cà Mau và được ký hiệu lần lượt từ $\mathrm{CM} 1$ đến $\mathrm{CM} 8$. Vị trí tọa độ của các ao từ $10^{\circ} 2^{\prime}-10^{\circ} 09^{\prime \prime} \mathrm{N}, 106^{\circ} 46^{\prime}-107^{\circ} 00^{\prime \prime} \mathrm{E}$ trong rừng ngập mặn Cà Mau (Hình 1). Tổng cộng có 3 đợt khảo sát: đợt tháng 3 (mùa khô $-\mathrm{K}$ ), tháng 7 (chuyển mùa $-\mathrm{C}$ ) và tháng 11 (mùa mưa - M) năm 2015.

\subsection{Phưong pháp thu mẫu}

Nồng độ oxy hòa tan (DO) được đo tại hiện trường bằng thiết bị $\mathrm{TOA}(\mathrm{WQC}-22 \mathrm{~A}, \mathrm{DKK}$ TOA Corporation, Tokyo, Japan), mỗi ao đo 3 mẫu lặp lại.

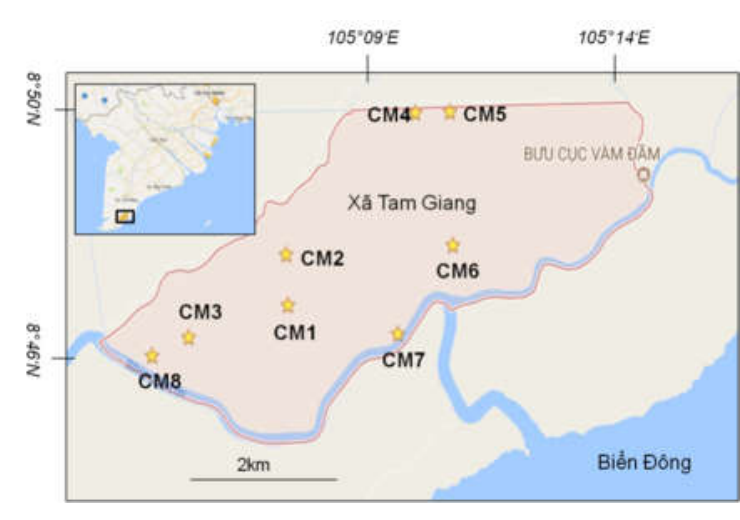

Hình 1. Sơ đồ các vị trí khảo sát

Tuyến trùng được thu bằng ống core (dài 30 $\mathrm{cm}$, đường kính $3,5 \mathrm{~cm}$ ) thu $10 \mathrm{~cm}$ lớp mặt (tương đương $10 \mathrm{~cm}^{2}$ trầm tích), mỗi ao thu 3 mẫu tuyến trùng. Sau đó cố định bằng dung dịch formaldehyde $10 \%$, nóng $60^{\circ} \mathrm{C}$ và chuyển về phòng thí nghiệm thuộc phòng Công nghệ và Quản lý Môi trường, Viện Sinh học Nhiệt đới để tiến hành phân tích.

\subsection{Phương pháp phân tích mẫu}

Mẫu tuyến trùng được lọc qua lưới $38 \mu \mathrm{m}$ và tách bằng phương pháp dùng dung dịch Ludox - TM50 (tỷ trọng 1,18) [10]. Sau đó mẫu được nhuộm với $3-5 \mathrm{ml}$ dung dịch Rose Bengal ( $1 \%$ ). Khoảng 100 cá thể (mẫu nào dưới 100 thì gắp toàn bộ) được gắp ngẫu nhiên để lên tiêu bản theo phương pháp của De Grisse (1969) [11]. Tuyến trùng được định danh đến giống nhờ các khóa phân loại của Warwick và cộng sự (1988) [12], Zullini (2005) [13], Nguyễn Vũ Thanh (2007) [14] và cơ sở dữ liệu tuyến trùng trực tuyến NeMys [15].

\subsection{Phuoong pháp xác định sinh khối và nhu cầu oxy của quần xã tuyến trùng}

Dùng kính hiển vi Optika B1000 BF có kết nối với một máy phân tích hình ảnh (Axiocam Zeiss), với chiều dài cơ thể $(\mathrm{L}, \mu \mathrm{m})$ được đo từ đầu đến đuôi (trừ đuôi filiform), chiều rộng cơ 
thể tuyến trùng $(\mathrm{W}, \mu \mathrm{m})$ được đo ở phần dày nhất của cơ thể (Platt và Warwick, 1983) [16].

Từ số liệu kích thước của tuyến trùng, sinh khối ướt $(\mu \mathrm{g})$ của từng cá thể được tính theo công thức của Andrassy (1956) [17]:

Sinh khối ướt $=\mathrm{L} \times \frac{W^{2}}{1600000}$

Trong đó: $L$ là chiều dài cơ thể lớn nhất của tuyến trùng $(\mu m)$

$\mathrm{Ox}=\frac{\left.\left(0.0449 * \sinh k h \hat{o ̂}_{i} k h \hat{o}^{\wedge} 0.8544 * \exp (\ln (Q 10) / 10) *(T-20)\right)\right) * 10^{\wedge} 9}{400000}$

(Ghi chú: 0,4 $\mathrm{gC}$ trao đổi chất sẽ tiêu tốn 1 lít $\mathrm{O}_{2}[20]$ )

\subsection{Phương pháp xư lý số liệu}

Để nêu lên sự khác biệt của các giá trị theo mùa, ao, phân tích ANOVA 2 nhân tố (two way ANOVA) được thực hiện, các giá trị $\mathrm{p}<$ 0,05 thì có ý nghĩa thống kê. Dùng phân mềm STATISTICA 7.0 để phân tích thống kê (số liệu được chuẩn hóa về dạng $\log (\mathrm{X}+1)$ trước khi phân tích). Ngoài ra, tương quan Pearson được dùng để phân tích tương quan giữa $\mathrm{DO}$ và nhu cầu oxy của QXTT.

\section{Kết quả và thảo luận}

3.1. Sinh khối quần xã tuyến trùng trong các ao tôm sinh thái qua 3 đọt khảo sát

Trong mùa khô, tổng sinh khối khô trung bình (SKKTB - $\mu \mathrm{gC} / 10 \mathrm{~cm}^{2}$ ) của QXTT dao động từ 25,55 (CM7) dến 225,43 (CM4). Ngoài ra, các ao $\mathrm{CM} 8,3,5$ và 6 cũng có sinh khối cao $(170,35$; 146,$53 ; 140,05$ và 140,80 ; tương ứng). Ao CM2 cũng ghi nhận sinh thối thấp $(51,22)$. Các giống Daptonema, Dichromadora, Parodontophora, Pomponema, Pseudolella, Ptycholaimellus, Sabatieria, Sphaerolaimus và Terschellingia có sinh khối cao trong quần xã (Bảng 1).

Sang chuyển mùa, tổng sinh khối khô của QXTT $\left(\mu \mathrm{gC} / 10 \mathrm{~cm}^{2}\right)$ cao hơn hẳn mùa khô, ao CM6 thâp nhất cũng đạt 206,46. Các ao CM5, 4,2 và 8 có sinh khối rất cao $(803,62 ; 733,24$; 550,15 và 533,83 , tương ứng). Ao CM1, 3 và 7 có sinh khối thấp hơn, đạt từ 409,47 đến 474,95. Những giống Daptonema, Dichromadora, Eumorpholaimus,
W là đường kính cơ thể lớn nhất của tuyến trùng $(\mu m)$

Sinh khối khô $(\mu \mathrm{gC})$ của tuyến trùng được thiết lập dựa trên sinh khối ướt [18] với công thức:

Sinh khối khô $=0.125 \%$ * Sinh khối ướt

Nhu cầu oxy của từng cá thể $(\mathrm{Ox}$ $\mathrm{nlO}_{2} /$ ngày/cá thể)
Halichoanolaimus, Marylynnia, Parodontophora,

Pomponema, Pseudolella, Sphaerolaimus, Subsphaerolaimus, Terschellingia và Viscosia có ưu thế về sinh khối trong quần xã (Bảng 2).

Đến mùa mưa, sinh khối khô của QXTT $\left(\mu \mathrm{gC} / 10 \mathrm{~cm}^{2}\right)$ có phần thấp hơn chuyển mùa. Cá biệt, ao CM4 có sinh khối đạt rất cao, đến 937,04. Các ao còn lại sinh khối dao động từ 24,77 (CM5) đến 555,46 (CM8). Nhóm các ao CM6, 2, 1 và 7 cũng có sinh khối khá cao, từ 129,74 đến 376,24. Các giống Chromadorita, Daptonema, Desmodora, Dichromadora, Gomphionema, Halalaimus, Marylynnia, Parodontophora, Pseudolella, Ptycholaimellus, Sphaerolaimus, Sphaerotheristus và Terschellingia có sinh khối cao trong quần xã (Bảng 3).

Các giống như Daptonema, Sphaerolaimus, Terschellingia có ưu thế về sinh khối qua cả 3 đợt khảo sát. Kết quả phân tích ANOVA 2 nhân tố cho thấy tổng sinh khối khô trung bình có sự khác biệt ở các ao, các mùa và cả tương tác ao, mùa $(\mathrm{p}$ mùa $=0,0006, \mathrm{p}$ ao $=0,02, \mathrm{p}$ ao $*$ mùa $=0,04)$.

Giá trị SKKTB trong ao NTST cao hơn rất nhiều khi so với SKKTB ở vùng ôn đới và cận nhiệt đới (trừ trường hợp ghi nhận SKKTB đạt $49-7044 \mu \mathrm{g} / 10 \mathrm{~cm}^{2}$ tại cửa sông Oosterschelde [21]. Cửa Swartskop (Nam Phi) từ 0,1 đến 0,4 $\mu \mathrm{g} / 10 \mathrm{~cm}^{2}$ [22], cửa sông Western Scheldt, từ $0,03-4,58 \mu \mathrm{g} / 10 \mathrm{~cm}^{2}$ [23], SKKTB ở đầm lầy Bizerte, vùng cận nhiệt đới Tunisia từ 66,13 đến $829,29 \mu \mathrm{g} / 10 \mathrm{~cm}^{2}$ [8]. Tổng sinh khối khô trung bình trong ao NTST cũng cao hơn SKKTB của QXTT vùng cửa sông Mekong trong nghiên cứu của tác giả Ngô Xuân Quảng và cộng sự (2014) $\left(9,08-706,3 \mu \mathrm{g} / 10 \mathrm{~cm}^{2}\right)$ [24]. 
Bảng 1. Sinh khối (tỷ lệ \%) của các giống ưu thế và của quần xã tuyến trùng trong ao nuôi tôm sinh thái trong mùa khô

\begin{tabular}{lrrrrrrrr}
\hline \multirow{2}{*}{ Giống } & \multicolumn{10}{c}{ Ao } \\
\cline { 2 - 9 } & \multicolumn{1}{c}{ CM1 } & \multicolumn{1}{c}{ CM2 } & \multicolumn{1}{c}{ CM3 } & \multicolumn{1}{c}{ CM4 } & \multicolumn{1}{c}{ CM5 } & \multicolumn{1}{c}{ CM6 } & \multicolumn{1}{c}{ CM7 } & CM8 \\
\hline Daptonema & $13,66(12,9)$ & $4,92(9,6)$ & $8,38(5,7)$ & $25,2(11,1)$ & $42,46(30,3)$ & $6,33(4,4)$ & $2,38(9,3)$ & $19,96(11,7)$ \\
Dichromadora & $6,51(6,14)$ & $1,46(2,8)$ & $3,13(2,1)$ & $10,42(4,6)$ & $7,38(5,2)$ & $1,1(0,78)$ & $1,98(7,7)$ & $7,78(4,57)$ \\
Parodontophora & $4,4(4,15)$ & $2,48(4,8)$ & $0,96(0,6)$ & $7,04(3,1)$ & $3,72(2,6)$ & $10,7(7,6)$ & $0,92(3,5)$ & $10,81(6,3)$ \\
Pomponema & $14,3(13,5)$ & $0,08(0,1)$ & $1,91(1,3)$ & $13,4(5,9)$ & $6,4(4,57)$ & - & $0,06(0,2)$ & $0,18(0,1)$ \\
Pseudolella & $1,86(1,7)$ & $1,97(3,8)$ & $3,33(2,2)$ & $23(10,2)$ & $4,11(2,9)$ & $7,54(5,3)$ & - & $15,59(9,1)$ \\
Ptycholaimellus & $11,77(11,1)$ & $0,17(0,3)$ & $15,84(1)$ & $8,77(3,8)$ & $1,36(0,9)$ & $9,84(6,9)$ & $0,9(3,52)$ & $2,09(1,2)$ \\
Sabatieria & - & $4,11(8,0)$ & $37,41(2)$ & $20,03(8,8)$ & $8,4(6)$ & - & $6,05(23)$ & $4,94(2,9)$ \\
Sphaerolaimus & - & - & $2,37(1,6)$ & $20,41(9,0)$ & $4,69(3,3)$ & $6,68(4,7)$ & $0,57(2,2)$ & $20,86(12,2)$ \\
Terschellingia & $39,94(37,6)$ & $13,13(25,6)$ & $9,46(6,4)$ & $12,59(5,5)$ & $25,17(17,9)$ & $21,54(15)$ & $7,9(30,9)$ & $19,28(11,3)$ \\
Giống khác & $13,5(12,74)$ & $22,89(44,6)$ & $63,74(43,5)$ & $84,58(37,5)$ & $36,38(25,9)$ & $77,08(54,7)$ & $4,8(18,7)$ & $68,86(40,4)$ \\
Tổng sinh khối & 105,94 & 51,22 & 146,53 & 225,43 & 140,05 & 140,80 & 25,55 & 170,35 \\
\hline
\end{tabular}

Ghi chú: - không xuất hiện 
Bảng 2. Sinh khối (tỷ lệ \%) của các giống ưu thế và của quần xã tuyến trùng trong ao nuôi tôm sinh thái thời điểm chuyển mùa

\begin{tabular}{|c|c|c|c|c|c|c|c|c|}
\hline \multirow{2}{*}{ Giống } & \multicolumn{8}{|l|}{ Ao } \\
\hline & CM1 & $\mathrm{CM} 2$ & CM3 & CM4 & CM5 & CM6 & CM7 & CM8 \\
\hline Daptonema & $22,78(5,6)$ & $67,48(12,2)$ & $91,32(18,8)$ & $94,82(12,9)$ & $125,95(15,6)$ & $20,49(9,9)$ & $13(2,74)$ & $70,86(13,2)$ \\
\hline Dichromadora & $15,33(3,7)$ & $55,59(10,1)$ & $33,06(6,8)$ & $29,68(4)$ & $34,02(4,2)$ & $3,14(1,5)$ & $91,15(19,1)$ & $5,5(1)$ \\
\hline Eumorpholaimus & $4,25(1)$ & $33,93(6,1)$ & $10,7(2,2)$ & - & $83,34(10,3)$ & $1,25(0,6)$ & $0,1(0,02)$ & $7,7(1,4)$ \\
\hline Halichoanolaimus & - & - & - & $120,83(16,4)$ & $43,49(5,4)$ & $2,23(1,08)$ & - & - \\
\hline Marylynnia & $4,77(1,1)$ & - & $42,13(8,6)$ & $10,42(1,4)$ & $22,14(2,7)$ & $11,78(5,7)$ & - & - \\
\hline Parodontophora & $9,7(2,3)$ & $15,82(2,8)$ & $14,77(3)$ & $8,82(1,2)$ & $17,67(2,2)$ & $3,27(1,5)$ & $26,44(5,5)$ & $24,87(4,6)$ \\
\hline Pomponema & $107,98(26,3)$ & $30,77(5,5)$ & $10,68(2,2)$ & $43,56(5,9)$ & $60,67(7,5)$ & $2,58(1,2)$ & - & $4,44(0,8)$ \\
\hline Pseudolella & $14,93(3,6)$ & $127,52(23,1)$ & $20,88(4,3)$ & $43,42(5,9)$ & $78,75(9,8)$ & $27,23(13,1)$ & $3,85(0,8)$ & $11,88(2,2)$ \\
\hline Sphaerolaimus & $105,81(25,8)$ & $3,64(0,6)$ & - & $37,07(5)$ & $58,98(7,3)$ & $57,17(27,6)$ & - & $20,38(3,8)$ \\
\hline Subsphaerolaimus & $10,19(2,4)$ & $35,23(6,4)$ & $104,33(21,5)$ & $15,35(2)$ & $2,15(0,2)$ & $31,38(15,2)$ & $190,81(40,1)$ & $10,19(1,9)$ \\
\hline Terschellingia & $42,51(10,3)$ & $60,12(10,9)$ & $24,97(5,1)$ & $233,07(31,7)$ & $44,74(5,5)$ & $11,67(5,6)$ & $124,68(26,2)$ & $62,35(11,6)$ \\
\hline Viscosia & $20,36(4,9)$ & $8,26(1,5)$ & $0,54(0,1)$ & $11,97(1,6)$ & $50,17(6,2)$ & $1,08(0,5)$ & $0,07(0,01)$ & - \\
\hline Giống khác & $50,87(12,4)$ & $111,79(20,3)$ & $131,49(27,1)$ & $84,23(11,4)$ & $181,56(22,5)$ & $33,19(16)$ & $24,86(5,2)$ & $315,66(59,1)$ \\
\hline Tổng sinh khối & 409,47 & 550,15 & 484,87 & 733,24 & 803,62 & 206,46 & 474,95 & 533,83 \\
\hline
\end{tabular}


Bảng 3. Sinh khối (tỷ lệ \%) của các giống ưu thế và của quần xã tuyến trùng trong ao nuôi tôm sinh thái trong mùa mưa

\begin{tabular}{|c|c|c|c|c|c|c|c|c|}
\hline \multirow{2}{*}{ Giống } & \multicolumn{8}{|c|}{ Ao } \\
\hline & CM1 & $\mathrm{CM} 2$ & CM3 & CM4 & CM5 & CM6 & CM7 & CM8 \\
\hline Chromadorita & $1,37(1)$ & - & - & $95,38(10,1)$ & $0,01(0,04)$ & $0,02(0,01)$ & $0,6(0,4)$ & $2,73(0,4)$ \\
\hline Daptonema & $9,26(6,7)$ & $28,71(13,1)$ & $7,08(21,6)$ & $175,5(18,7)$ & $2,54(10,2)$ & $10,07(2,6)$ & $7,25(5,5)$ & $11,55(2,08)$ \\
\hline Desmodora & $4,47(3,2)$ & $8,75(3,9)$ & $0,06(0,1)$ & $68,75(7,3)$ & $0,08(0,3)$ & $5,11(1,36)$ & $0,66(0,51)$ & $1,11(0,2)$ \\
\hline Dichromadora & $30,94(22,4)$ & $4,11(1,8)$ & $0,37(1,1)$ & $55,7(5,9)$ & $0,61(2,4)$ & $12,62(3,3)$ & $28,88(22,2)$ & $42,83(7,7)$ \\
\hline Gomphionema & $2,44(1,7)$ & $7,46(3,4)$ & $1,43(4,3)$ & $28,19(3)$ & $0,76(3)$ & $0,39(0,1)$ & $8,54(6,5)$ & $9,23(1,6)$ \\
\hline Halalaimus & $2,24(1,6)$ & $0,09(0,04)$ & $0,54(1,6)$ & $1,59(0,17)$ & $0,08(0,33)$ & $17,49(4,6)$ & - & $49,32(8,8)$ \\
\hline Marylynnia & - & - & - & - & - & $29,11(7,7)$ & - & $14,51(2,6)$ \\
\hline Parodontophora & $6,42(4,6)$ & $11,33(5,1)$ & $1,51(4,6)$ & $54,56(5,8)$ & $0,5(2,02)$ & $11,58(3,08)$ & $4,95(3,8)$ & $24,88(4,4)$ \\
\hline Pseudolella & $14,4(10,4)$ & $19,26(8,7)$ & $2,89(8,8)$ & $27,18(2,9)$ & $0,79(3,1)$ & $8,66(2,3)$ & $5,61(4,3)$ & $33,83(6,09)$ \\
\hline Ptycholaimellus & $0,14(0,1)$ & $1,62(0,7)$ & $0,37(1,1)$ & $95,3(10,1)$ & $0,27(1,1)$ & $6,74(1,7)$ & $0,05(0,04)$ & $0,24(0,04)$ \\
\hline Sphaerolaimus & $2,2(1,6)$ & $8,79(4,01)$ & - & - & $0,59(2,3)$ & $34,19(9,09)$ & $9,46(7,2)$ & $39,72(7,1)$ \\
\hline Sphaerotheristus & $10,36(7,5)$ & $5,91(2,7)$ & $1,61(4,9)$ & $35,24(3,7)$ & $1,8(7,2)$ & $26,96(7,1)$ & $1,89(1,4)$ & $15,32(2,7)$ \\
\hline Terschellingia & $20,72(15,06)$ & $31,83(14,5)$ & $9,02(27,6)$ & $227,33(24,2)$ & $3,29(13,2)$ & $193,39(51,4)$ & $49,99(38,5)$ & $186,12(33,5)$ \\
\hline Giống khác & $32,6(23,7)$ & $91,17(41,6)$ & $7,79(23,8)$ & $72,35(7,7)$ & $13,45(54,2)$ & $19,93(5,3)$ & $11,85(9,1)$ & $124,08(22,3)$ \\
\hline Tổng sinh khối & 137,56 & 219,04 & 32,67 & 937,04 & 24,77 & 376,24 & 129,74 & 555,46 \\
\hline
\end{tabular}

Ghi chú: - không xuất hiện 


\subsection{Nhu cầu oxy của quần xã tuyến trùng}

Nồng độ oxy (DO - mg/l) ở các ao tôm dao động từ 4,50 (CM4) đến 11,27 (CM7) trong mùa khô, từ 5,96 (CM4) đến 9,83 (CM7) trong chuyển mùa và ở mùa mưa dao động từ 4,97 (CM4) đến 13,50 (CM7). Dễ thấy ao CM4 có DO thấp nhất, ngược lại ao $\mathrm{CM} 7$ có $\mathrm{DO}$ cao nhất trong 3 đợt khảo sát. Phân tích ANOVA 2 nhân tố cho thấy DO có sự khác biệt ở các ao, các mùa và cả tương tác ao, mùa ( $\mathrm{p}$ mùa, $\mathrm{ao}$, ao * mùa $<0,05)($ Hình 2A).

Nhu cầu oxy $\left(\mathrm{Ox}-\mathrm{nlO} 2 / \mathrm{ngày} / 10 \mathrm{~cm}^{2}\right)$ của QXTT trong mùa khô đạt từ 3767,38 (CM7) đến 20654,03 (CM4). Các ao CM8, 5, 3 và 6 cũng có Ox cao $(19149,36 ; 13989,39 ; 16405,89$ và 16475,51 , tương ứng). Ao $\mathrm{CM} 1,2$ có $\mathrm{Ox}$ thấp, chỉ đạt 11487,45 và 6751,71 , tương ứng. Sang mùa khô, Ox tăng lên rất cao (đạt đến
57355,93 - CM5). Ngoài ra các ao CM4, 1, 2, 3, 7 và 8 cũng có $O x$ cao, dao động từ 32559,55 đến 50734,69. Ở mùa mưa, ao CM4 ghi nhận Ox rất cao (đạt đến 64228,47 ). Các ao còn lại dao động từ 3467,39 (CM3) đến 49239,51 (CM8) (Hình 2B). Kết quả phân tích ANOVA 2 nhân tố cho thấy Ox có sự khác biệt ở các ao, các mùa và cả tương tác $\mathrm{ao}$, mùa $(\mathrm{p}$ mùa $=$ $0,0006, p$ ao $=0,03, p$ ao $*$ mùa $=0,04)$.

Có rất ít nghiên cứu về nhu cầu oxy của QXTT trên thế giới và Việt Nam, trong khi đây là một trong những vấn đề quan trọng trong nghiên cứu về sinh thái tuyến trùng. Năm 2007, tác giả Boufahja và cộng sự (2007) đã nghiên cứu $\mathrm{Ox}$ của $\mathrm{QXTT}$ tại vùng đầm lầy Bizerte, vùng cận nhiệt đới Tunisia, từ 5310 đến $43180 \mathrm{nlO} 2 /$ ngày $/ 10 \mathrm{~cm}^{2}$ [8], giá trị $\mathrm{Ox}$ này thấp hơn $\mathrm{Ox}$ trong ao NTST trong rừng ngập mặn Cà Mau.

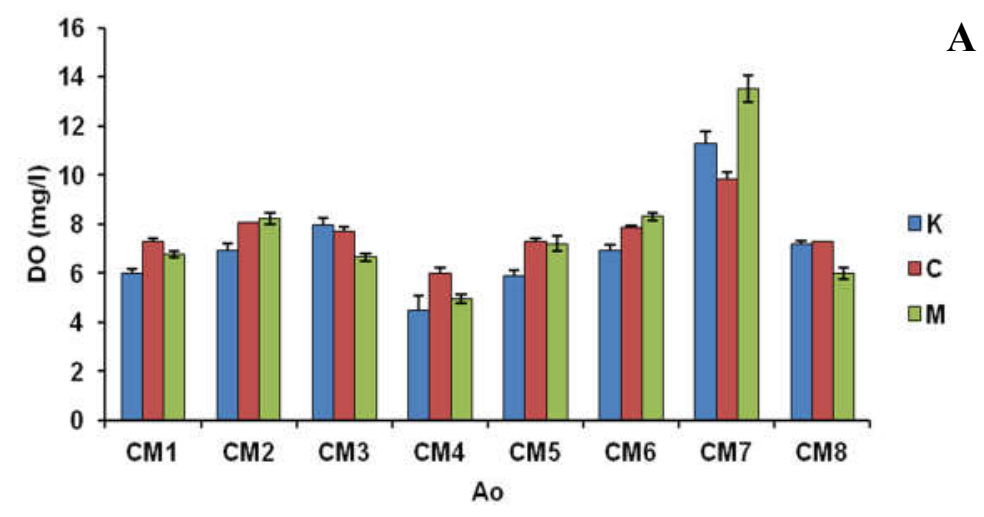

B

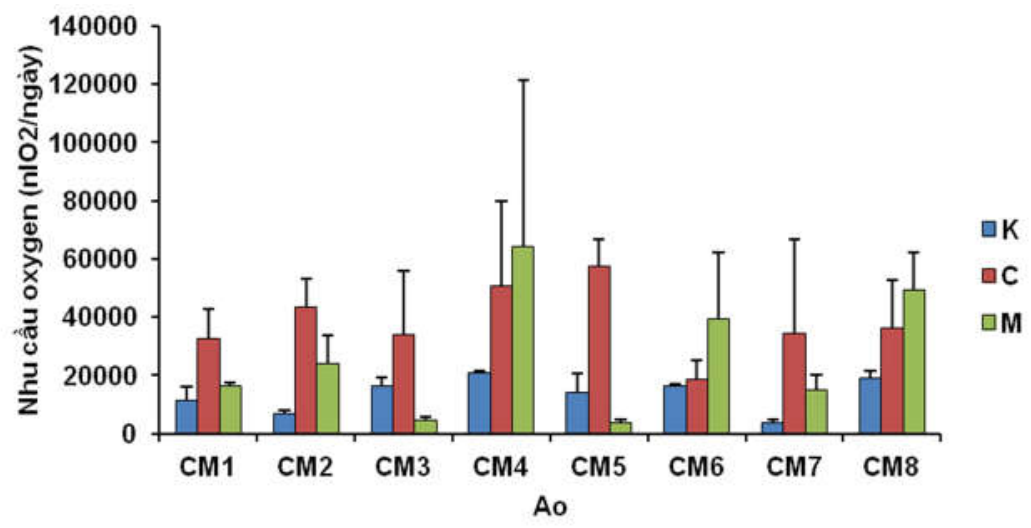

Hình 2. Nồng độ oxy hòa tan $(\mathrm{A})$ và nhu cầu oxy của quần xã tuyến trùng $(\mathrm{B})$. 
3.3. Tưong quan giữa nồng độ oxy hòa tan và nhu cầu oxy của quần xã tuyến trùng

Kết quả phân tích tương quan Pearson cho thấy $\mathrm{DO}$ và $\mathrm{Ox}$ tương quan nghịch khá cao và có ý nghĩa thống kê. Cụ thể, trong mùa khô giá trị $\mathrm{r}=-0,48, \mathrm{p}=0,03$, chuyển mùa $\mathrm{r}=-0,44, \mathrm{p}$ $=0,03$. Tuy nhiên, mối tương quan này không có ý nghĩa thống kê vào mùa mưa $(r=-0,09, p$ $=0,66$ ). Các mô hình tương quan được thể hiện trong Hình 3.

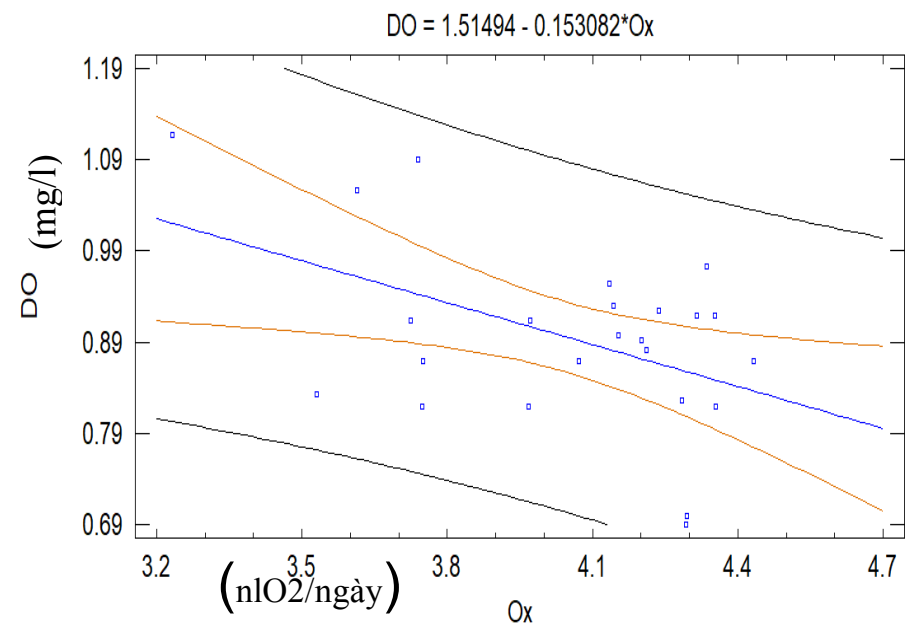

A

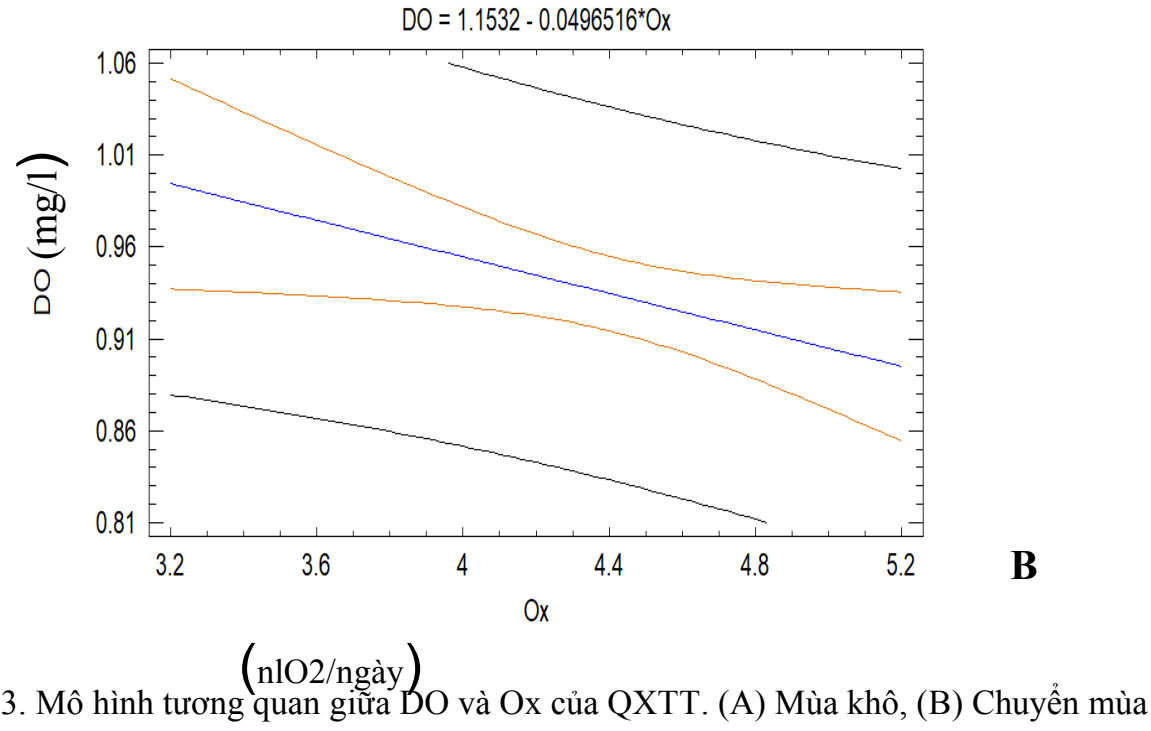

Tương quan nghịch giữa $\mathrm{Ox}$ và $\mathrm{DO}$ cho thấy khi nhu cầu trao đổi chất của QXTT tăng sẽ làm tăng nhu cầu oxy, từ đó làm giảm lượng oxy hòa tan trong ao. Dễ thấy qua 3 đợt khảo sát, mặc dù $\mathrm{DO}$ vẫn nằm trong giới hạn cho phép (từ $4-15 \mathrm{mg} / \mathrm{l}$ ) [25] nhưng hầu hết tập trung ở khoảng $7-9 \mathrm{mg} / \mathrm{l}$ và có giá trị nhỏ hơn
$4 \mathrm{mg} / 1$ (Hình 4). Như vậy có thể thấy DO tương đối thấp. Có nhiều nhóm sinh vật tiêu thụ oxy trong ao tôm, nhóm sinh vật đáy chỉ là một trong số đó. Quá trình hô hấp, trao đổi chất của nhóm sinh vật đáy (cụ thể là nhóm tuyến trùng) có tương quan nghịch với $\mathrm{DO}$ lần đầu tiên được ghi nhận trong nghiên cứu này. 


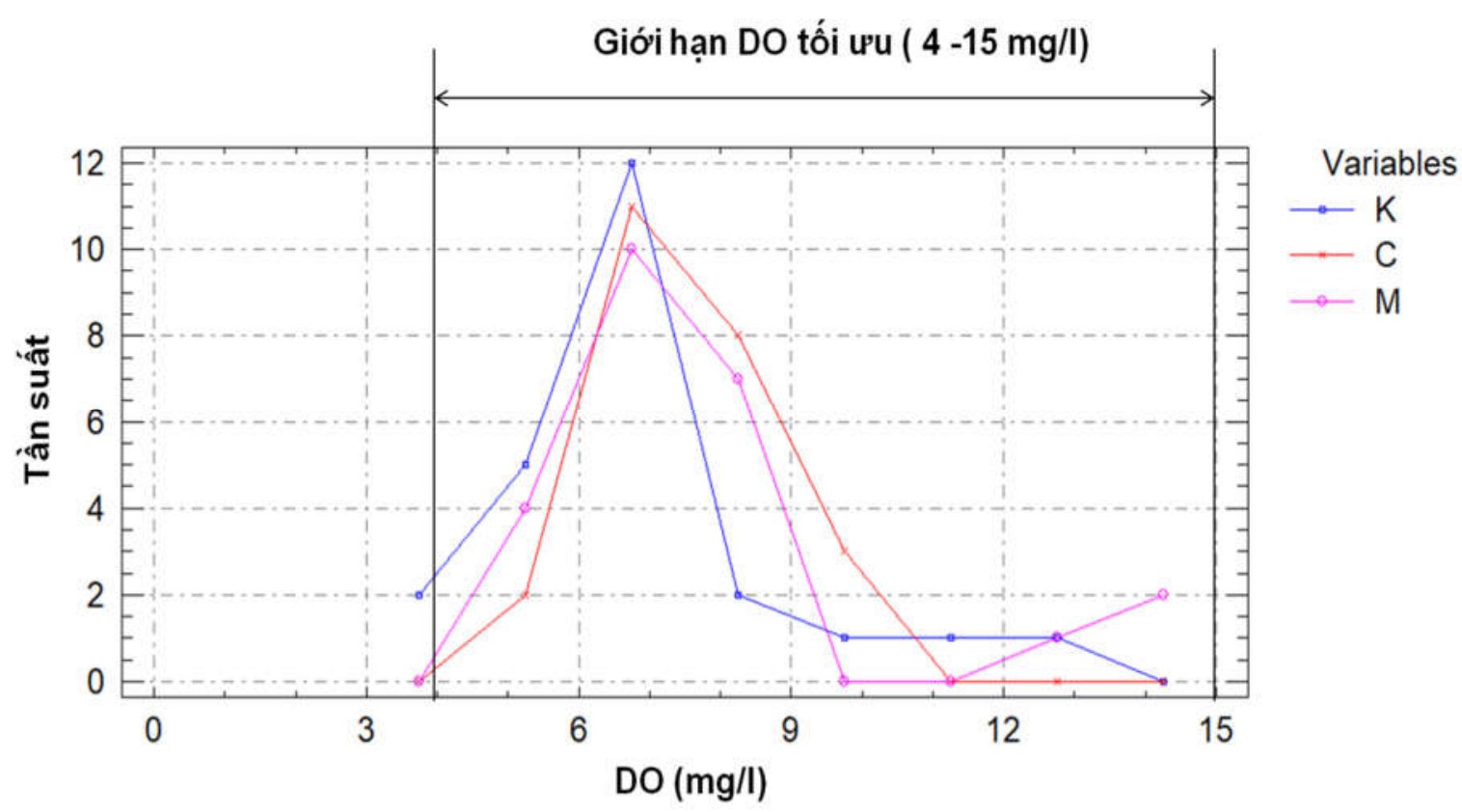

Hình 4. Phân phối tuần suất DO ở 3 mùa khảo sát

\section{Kết luận}

Kết quả ngiên cứu ghi nhận mối tương quan nghịch có ý nghĩa thống kê giữa $\mathrm{DO}$ và $\mathrm{Ox}$ của QXTT trong các ao NTST xã Tam Giang, huyện Năm Căn, tỉnh Cà Mau. Ngoài ra, SKKTB và $\mathrm{Ox}$ của $\mathrm{QXTT}$ ở ao NTST trong rừng ngập mặn Cà Mau cũng cao hơn so với các nghiên cứu khác trên thế giới. Nghiên cứu cũng cho thấy, mặc dù $\mathrm{DO}$ vẫn nằm trong giới hạn cho phép như hầu hết giá trị đều tập trung ở giới hạn dưới.

\section{Tài liệu tham khảo}

[1] P. N. Hong, H. T. San, Mangroves of Vietnam 7 (1993) IUCN.

[2] T. Nga, Hệ thống rừng - tôm trong phát triển bền vững vùng ven biển đồng bằng sông Cửu Long. Tạp chí Khoa học Trường Đại học Cần Tho 10 (2008) 6.

[3] Thai agricutural standard (TAS), Organic marine shrimp farming, Royal Gazette 124 (2007) Section 78E.
[4] T. T. Thai, N. T. My Yen, N. Tho, N. X. Quang, Meiofauna in the mangrove-shrimp farms ponds, $\mathrm{Ca} \mathrm{Mau}$ province. Journal of Science and Technology 55(2017) 271.

[5] L. Marte, The Food and Feeding Habit of Penaeus Monodon Fabricius Collected From Makato River, Aklan, Philippines (Decapoda Natantia) 1, Crustaceana 38(1980) 225.

[6] N. Majdi, W. Traunspurger, Free - living nematodes in the freshwater food web: a review, Journal of nematology 47 (2015) 28.

[7] M. C. Austen, Natural nematode communities are useful tools to address ecological and applied questions, Nematology Monographs and Perspectives 2 (2004) 1.

[8] F. Boufahja, H. Beyrem, N. Essid, J. Amorri, E. Mahmoudi, P. Aissa, Morphometry, energetics and diversity of free-living nematodes from coasts of Bizerte lagoon (Tunisia): an ecological meaning, Cahiers de biologie marine 48 (2007) 121.

[9] Ministry of Agriculture and Rural development, $2016 . \quad \mathrm{https}: / /$ tongcucthuysan.gov.vn/enus/aquaculture/doc-tin/006222/2016-10-28/camau-set-outs-to-become-viet-nams-largestshrimp-hub. Truy cập ngày 14/8/2017.

[10] M. Vincx, Meiofauna in marine and freshwater sediments, In G. S. Hall (Ed.), Methods for the 
examination of organismal diversity in soils and sediments Wallinfort, UK, 1996.

[11] A. T. De Grisse, Redescription ou modifications de quelques technique utilis [a] es dan l'etude des $\mathrm{n}$ [a] ematodes phytoparasitaires (1969).

[12] R.M. Warwick, H.M. Platt, P.J. Somerfield, Free living marine nematodes. Part III. Monhysterids. The Linnean Society of London and the Estuarine and Coastal Sciences Association, London 1988.

[13] Zullini, The Identification manual for freshwater nematode genera, Lecture book, MSc Nematology Ghent University 2005.

[14] N. V. Thanh, Giun tròn sống tự do Monhysterida, Araeolaimida, Chromadorida, Rhabditida, Enoplida, Mononchida, Dorylaimida. Động vật chí Việt Nam. Hà Nội: Nhà xuât bản khoa học và kỹ thuật, 22, 2007455.

[15] J. Vanaverbeke, T.N. Bezerra, U. Braeckman, A. De Groote, N. De Meester, T. Deprez, S. Derycke, K. Guilini, F. Hauquier, L. Lins, T. Maria, T. Moens, E. Pape, N. Smol, , M. Taheri, J. Van Campenhout, A. Vanreusel, X. Wu, M. Vincx, (2015)

[16] NeMys: World Database of Free - Living Marine Nematodes. Accessed at http://nemys.ugent.be on 2017.

[17] H. M. Platt, R. M. Warwick, Freeliving marine nematodes. Part 1: British enoplids. Pictorial key to world genera and notes for the identification of British species. Cambridge University Press, for the Linnean Society of London and the Estuarine and Brackish-water Sciences Association 1983.

[18] Andrassy I The determination of volume and weight of nematodes, Acta Zoologica 2 (1956) 1.

[19] J. Vanaverbeke, P. M. Arbizu, H. U. Dahms, H. K. Schminke,. The metazoan meiobenthos along a depth gradient in the Arctic Laptev Sea with special attention to nematode communities, Polar Biology 18 (1997) 391.

[20] K. Soetaert, J. Vanaverbeke, C. Heip, P. M. Herman, J. J. Middelburg, A. Sandee, G. Duineveld, Nematode distribution in ocean margin sediments of the Goban Spur (northeast Atlantic) in relation to sediment geochemistry, Deep Sea Research Part I: Oceanographic Research Papers, 44 (1997) 1671.

[21] D.J. Crisp Methods of the study of marine benthos (N.A. Holme \& A.D. McIntyre eds), Blackwell Scientific Publications, Oxford, 1971197.

[22] N. Smol, K. A. Willems, J. C. Govaere, A. J. J. Sandee, Composition, distribution and biomass of meiobenthos in the Oosterschelde estuary (SW Netherlands). In The Oosterschelde Estuary (The Netherlands): a Case-Study of a Changing Ecosystem, Springer Netherlands (1994) 197.

[23] H. Dye, An Ecophysiological Study of the Meiofauna of the Swartkops Estuary, African Zoology 13(1978) 1.

[24] Van Damme, R. Herman, Y. Sharma, M. Holvoet, P. Martens, Benthic studies of the Southern Bight of the North Sea and its adjacent continental estuaries, Progress Report II. Fluctuations of the meiobenthic communities in the Westerschelde estuary. ICES. CM/L, 23 (1980) 131.

[25] Q. X. Ngo, C. Nguyen Ngoc, A. Vanreusel, Nematode morphometry and biomass patterns in relation to community characteristics and environmental variables in the Mekong Delta, Vietnam, Raffles Bulletin of Zoology 62 (2014) 501.

[26] J. M. Whetstone, G. D. Treece, C. L. Browdy, A. D. Stokes, Opportunities and constraints in marine shrimp farming, South Regional Aquaculture Center 2002. 


\title{
Correlation between Oxygen Demand of Nematode Communities with Dissolved Oxygen in the Organic Shrimp Farming Ponds, Ca Mau Province
}

\author{
Tran Thanh Thai ${ }^{1}$, Nguyen Le Que Lam ${ }^{1}$, \\ Nguyen Thi My Yen ${ }^{1,2}$, Ngo Xuan Quang ${ }^{1}$ \\ ${ }^{1}$ Institute of Tropical Biology, Vietnamese Academy of Science and Technology \\ 85 Tran Quoc Toan Street, Ho Chi Minh City, Vietnam \\ ${ }^{2}$ Ghent University, 281 Krijgslaan, S8, B - 9000 Ghent, Belgium
}

\begin{abstract}
Dissolved oxygen (DO), total biomass and oxygen demand of nematode communities in the organic shrimp farms located in Tam Giang commune, Nam Can District, Ca Mau province were investigated in three seasons (March-dry, July-transfer and November-rain season) of 2015. The results showed that most of DO values were within permissible limits. However, the frequency distributions of DO values are very compressed at the lower limit of their scale. Total dry biomass varied from 24.77 to $937.04 \mu \mathrm{gC} / 10 \mathrm{~cm}^{2}$ while oxygen demand ranged from 3467.39 to 64288.50 $\mathrm{nlO}_{2} /$ day $/ 10 \mathrm{~cm}^{2}$. These values were slightly high when compared to other studies in the world. The following results recorded that the negatively correlation between DO and oxygen demand of nematode communities in the organic shrimp farms. This may well suggest that respiration and metabolic of nematode communities was high and their impact on oxygen dissolved in surface water.
\end{abstract}

Keywords: Biomass, dissolved oxygen, Ca Mau, nematode communities, organic shrimp farms, oxgen demand 